\title{
Internet of Things (IOT)-Based Behavioral Intervention for Older Adults with Major Depressive Disorder: Preliminary study
}

Sang Joon Son,MD, $\mathrm{PhD}^{1}{ }^{1}$ *; Chang Hyung Hong, MD, PhD¹; Jae Hoon Kim, PhD2; Ki Young Lim, MD, PhD ${ }^{1,}$ *

${ }^{1}$ Department of Psychiatry, Ajou University School of Medicine, Suwon, Republic of Korea

2 Department of Industrial Engineering, Ajou University, Suwon, Republic of Korea

Objective: To assess the effectiveness of Internet of Things (loT)-based behavioral intervention for reducing depressive symptom of older adults with major depressive disorder.

Method: A 12-week randomized cross-over controlled study was conducted at community mental health center. We recruited 39 participants with major depressive disorder aged 60 years or older. As a multidomain intervention, four evidence-based therapeutic factors (physical activity, healthy diet, social activity, and emotional regulation) were approached. To maintain motivation of participants, we applied contingency management using loT device based on operant conditioning theory.

Results: The primary outcome was change of depressive symptom measured by Montgomery-Asberg Depression Rating Scale (MADRS). Mixed-effect model compared the effectiveness of intervention and usual care management (intervention by time and period interaction, $p=0.017$ )

Conclusions: Community-implementable loT-based behavioral intervention resulted in greater reduction of depressive symptom of elderly with major depressive disorder.

Keywords: Depression, Elderly, Internet of Things, Psychosocial intervention

ACKNOWLEDGMENTS: This study was supported by a grant from the Korea Health 21 R\&D Project, Ministry of Health \& Welfare, Republic of Korea (HI15C0995)

Figure 1. Flow diagram for 'Internet of things gold medal program'
Table 1. Baseline characteristics of group 1 versus group 2, completers versus dropouts

\begin{tabular}{|c|c|c|c|c|c|c|c|c|}
\hline Variable & $\begin{array}{c}\text { Group 1 } \\
\text { (Intervention } \rightarrow \text { Control) } \\
(n=20)\end{array}$ & $\begin{array}{c}\text { Group } 2 \\
(\text { Control } \rightarrow \text { Intervention }) \\
(n=19)\end{array}$ & $x^{2}$ ort & $\mathrm{p}$-value & $\begin{array}{c}\text { Completers } \\
(n=35)\end{array}$ & $\begin{array}{l}\text { Dropouts } \\
(n=4)\end{array}$ & $t$ & $\mathrm{p}$-value \\
\hline Age (years) & $73.9 \pm 5.6$ & $73.4 \pm 6.1$ & 0.2 & 0.820 & $73.9 \pm 5.5$ & $71.3 \pm 8.4$ & 0.874 & 0.388 \\
\hline Sex (female) & $14(70.0)$ & $16(84.2)$ & 1.1 & 0.292 & $27(77.1)$ & $3(75.0)$ & & 1.000 \\
\hline Years of education & $9.5 \pm 5.3$ & $7.1 \pm 3.9$ & 1.6 & 0.119 & $8.2 \pm 4.9$ & $9.0 \pm 3.5$ & 0.300 & 0.766 \\
\hline MADRS & $29.0 \pm 10.0$ & $31.2 \pm 9.1$ & 0.7 & 0.485 & $29.1 \pm 9.5$ & $38.3 \pm 3.8$ & 1.887 & 0.067 \\
\hline BAl & $18.1 \pm 10.9$ & $17.0 \pm 10.3$ & 0.3 & 0.760 & $17.6 \pm 10.7$ & $17.3 \pm 10.6$ & 0.057 & 0.955 \\
\hline K-MMSE & $25.7 \pm 2.7$ & $24.2 \pm 3.5$ & 1.5 & 0.143 & $24.7 \pm 3.2$ & $26.8 \pm 2.8$ & 1.210 & 0.234 \\
\hline
\end{tabular}

Table 2. Means and standard deviations of primary and secondary outcomes at baseline and follow-ups

\begin{tabular}{|c|c|c|c|c|c|c|}
\hline & \multicolumn{2}{|c|}{ MADRS } & \multicolumn{2}{|c|}{ BAI } & \multicolumn{2}{|c|}{ K-MMSE } \\
\hline & $\begin{array}{c}\text { Group } 1 \\
\text { Mean (SD) }\end{array}$ & $\begin{array}{c}\text { Group } 2 \\
\text { Mean (SD) }\end{array}$ & $\begin{array}{l}\text { Group } 1 \\
\text { Mean (SD) }\end{array}$ & $\begin{array}{c}\text { Group } 2 \\
\text { Mean (SD) }\end{array}$ & $\begin{array}{c}\text { Group } 1 \\
\text { Mean (SD) }\end{array}$ & $\begin{array}{c}\text { Group } 2 \\
\text { Mean (SD) }\end{array}$ \\
\hline Visit I (baseline) & $29.0(10.0)$ & $31.2(9.1)$ & $18.1(10.9)$ & $17.0(10.3)$ & $25.7(2.7)$ & $24.2(3.5)$ \\
\hline Visit 2 & $16.6(8.7)$ & $23.4(11.1)$ & $14.7(13.2)$ & $14.3(12.6)$ & & \\
\hline Visit 3 & $16.4(10.8)$ & $18.6(10.2)$ & $15.5(13.7)$ & $15.6(10.6)$ & & \\
\hline Visit 4 (washout) & $7.7(3.4)$ & 21.1 (11.5) & $12.9(12.0)$ & $9.4(8.8)$ & $26.6(2.4)$ & $24.3(4.0)$ \\
\hline Visit 5 & 17.1 (11.5) & $22.3(10.7)$ & $9.4(8.8)$ & $12.9(12.0)$ & $24.3(4.0)$ & $26.6(2.4)$ \\
\hline Visit 6 & $13.9(9.7)$ & $18.2(8.7)$ & $12.2(9.7)$ & $12.7(12.5)$ & & \\
\hline Visit 7 & $9.6(6.8)$ & $15.4(6.5)$ & $10.5(11.8)$ & $11.8(11.1)$ & & \\
\hline Visit 8 & $12.8(8.3)$ & $20.6(11.4)$ & $9.2(8.3)$ & $14.1(12.5)$ & $25.8(3.0)$ & $27.0(2.9)$ \\
\hline
\end{tabular}

Figure 2. MADRS at each time measure compared with baseline performance

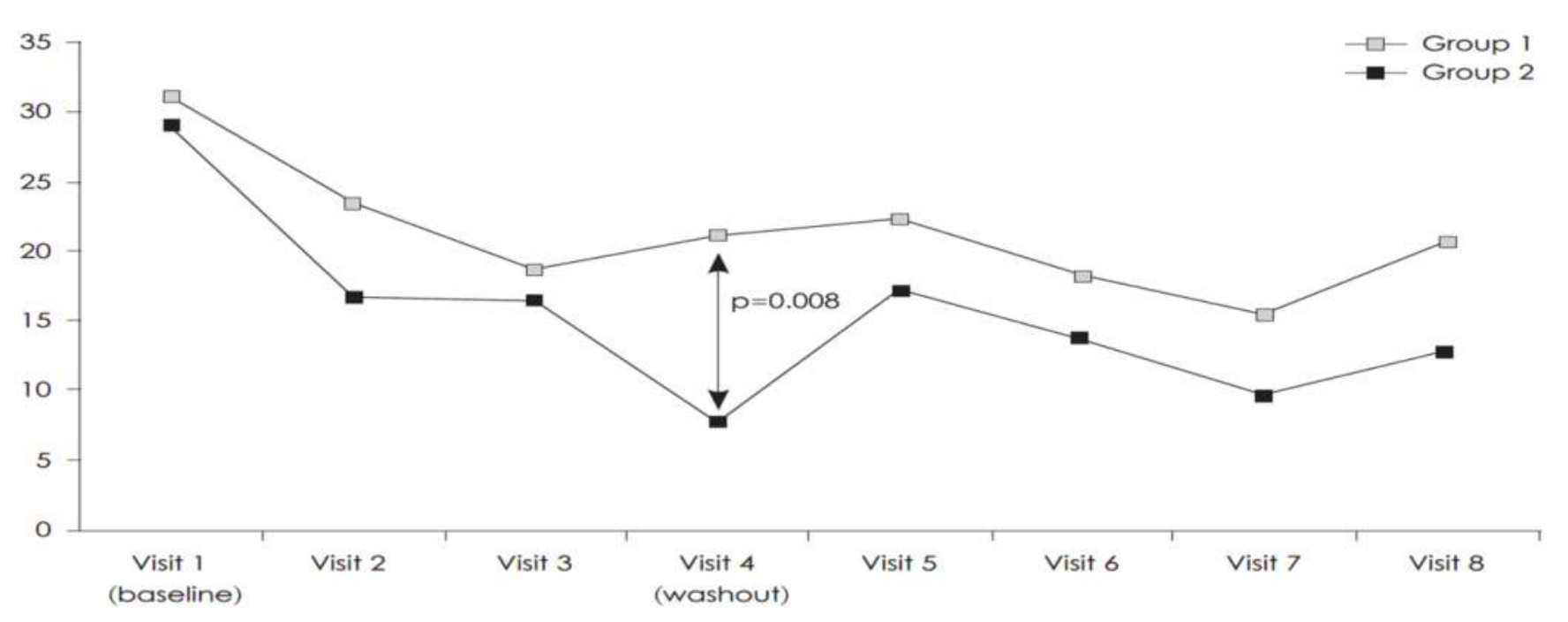

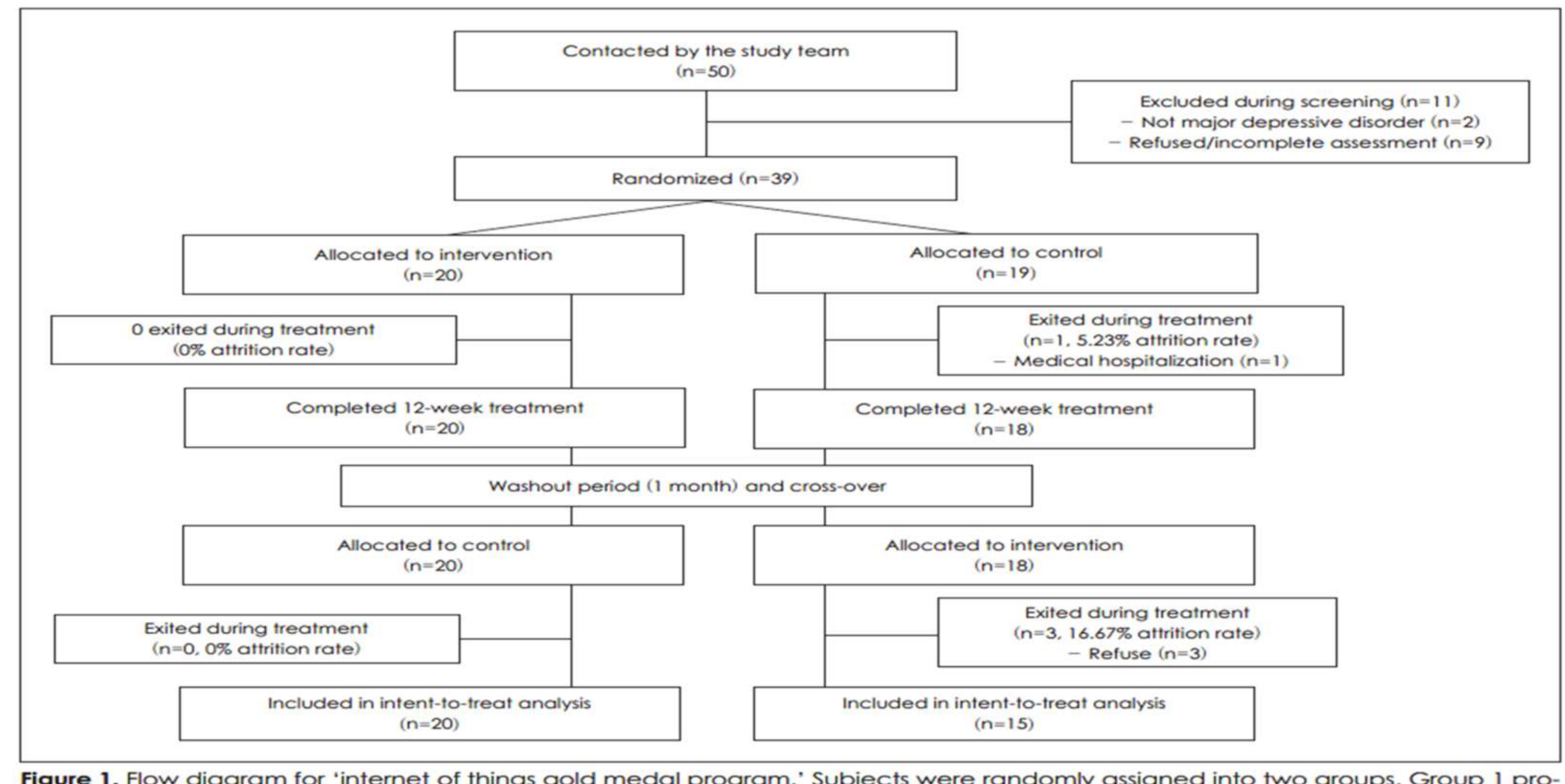

